Brief note

\title{
EXPERIMENTAL AND ANALYTICAL RESEARCH ON RESONANCE PHENOMENA OF VIBRATING HEAD WITH MRE REGULATING ELEMENT
}

\author{
D. MIEDZIŃSKA* and R. GIELETA \\ Military University of Technology \\ Department of Mechanics and Applied Computer Science \\ 2 Gen. S. Kaliskiego St., 00-908 Warsaw, POLAND \\ E-mails: dmiedzinska@wat.edu.pl \\ rgieleta@wat.edu.pl

\section{J. OSIŃSKI} \\ Warsaw University of Technology \\ Faculty of Automotive and Construction Machinery Engineering \\ 84 Narbutta St., 02-524 Warsaw, POLAND \\ E-mail: josinski@ipbm.simr.pw.edu.pl
}

\begin{abstract}
A vibratory pile hammer (VPH) is a mechanical device used to drive steel piles as well as tube piles into soil to provide foundation support for buildings or other structures. In order to increase the stability and the efficiency of the VPH work in the over-resonance frequency, a new VPH construction was developed at the Military University of Technology. The new VPH contains a system of counter-rotating eccentric weights, powered by hydraulic motors, and designed in such a way that horizontal vibrations cancel out, while vertical vibrations are transmitted into the pile. This system is suspended in the static parts by the adaptive variable stiffness pillows based on a smart material, magnetorheological elastomer (MRE), whose rheological and mechanical properties can be reversibly and rapidly controlled by an external magnetic field. The work presented in the paper is a part of the modified VPH construction design process. It concerns the experimental research on the vibrations during the piling process and the analytical analyses of the gained signal. The results will be applied in the VPH control system.
\end{abstract}

Key words: vibratory pile hammer, resonance, vibrations, Fourier analysis.

\section{Introduction}

A vibratory pile hammer (VPH) is a mechanical device used to drive steel piles as well as tube piles into soil to provide foundation support for buildings or other structures. In order to increase the stability and the efficiency of the VPH work in the over-resonance frequency, a new VPH construction was developed at the Military University of Technology. The new VPH contains a system of counter-rotating eccentric weights, powered by hydraulic motors, and designed in such a way that horizontal vibrations cancel out, while vertical vibrations are transmitted into the pile. This system is suspended in the static parts by the adaptive variable stiffness pillows based on a smart material, magnetorheological elastomer (MRE), whose rheological and mechanical properties can be reversibly and rapidly controlled by an external magnetic field (An and Shaw, 2003).

\footnotetext{
" To whom correspondence should be addressed
} 
The vibration head that will be modified with the MRE regulation system is presented in Fig.1. The elastomer pillows (number 7 in Fig.2) will be enhanced with the MRE and the regulation system (coils).

Magnetorheological elastomers (MREs) belong to the group of so-called smart materials, which respond to an external stimulus by changing their viscoelastic properties. They are the solid analogues of magnetorheological fluids (MRFs), consisting of magnetically permeable particles (such as iron) added to a viscoelastic polymeric material prior to crosslinking (Kallio et al., 2003; Boczkowska and Awietjan, 2009). Before the curing process of the polymer a strong external magnetic field is applied. The field induces dipole moments within the particles, which seek minimum energy states. The chains of particles with collinear dipole moments are formed and curing of the polymeric matrix material locks the chains in place. In this orientation, the particles can form separate chains in the three-dimensional simple lattice structures or even more complicated structures, where particles have multiple interaction points (An and Shaw, 2003; Boczkowska and Awietjan, 2009). The microstructure of the MRE cured under the magnetic field is presented in Fig.3.

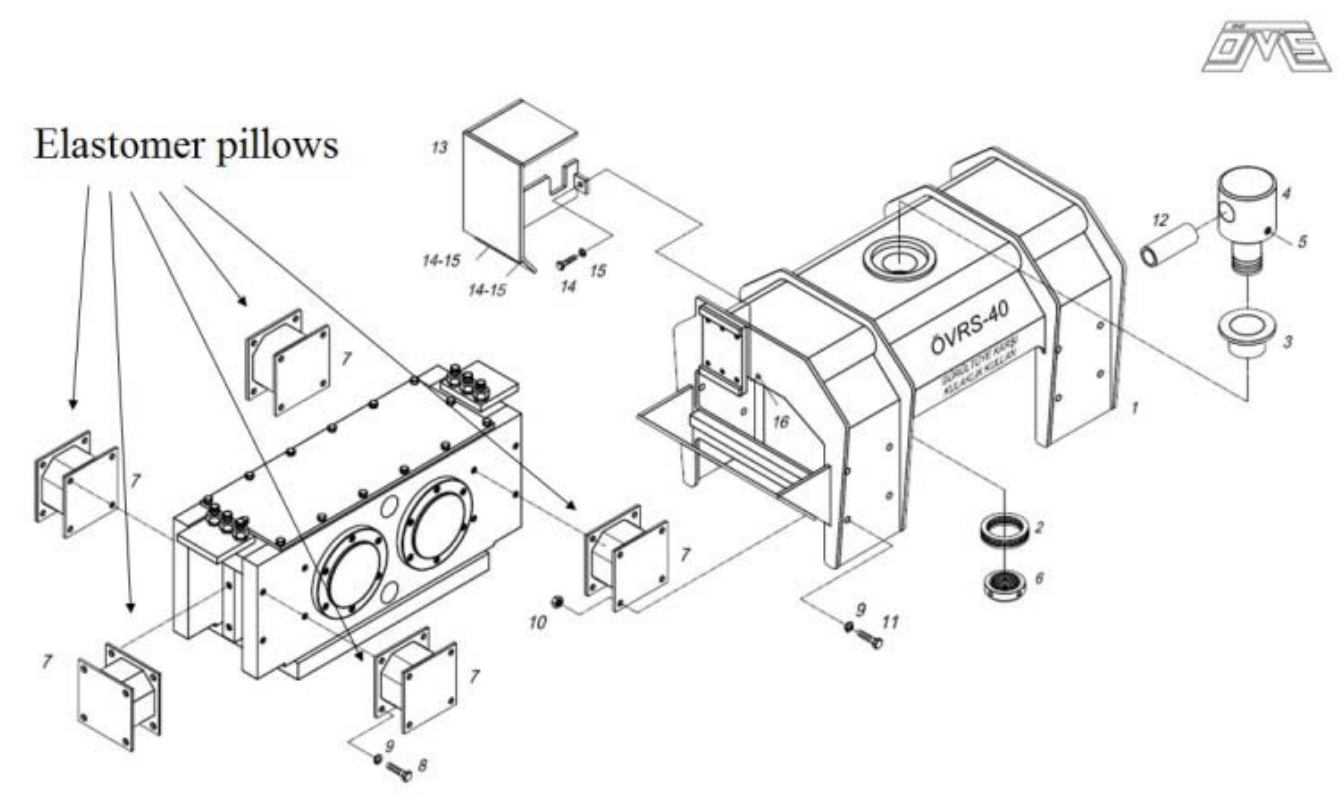

Fig.1. Inside construction of VPH with elastomer pillows.
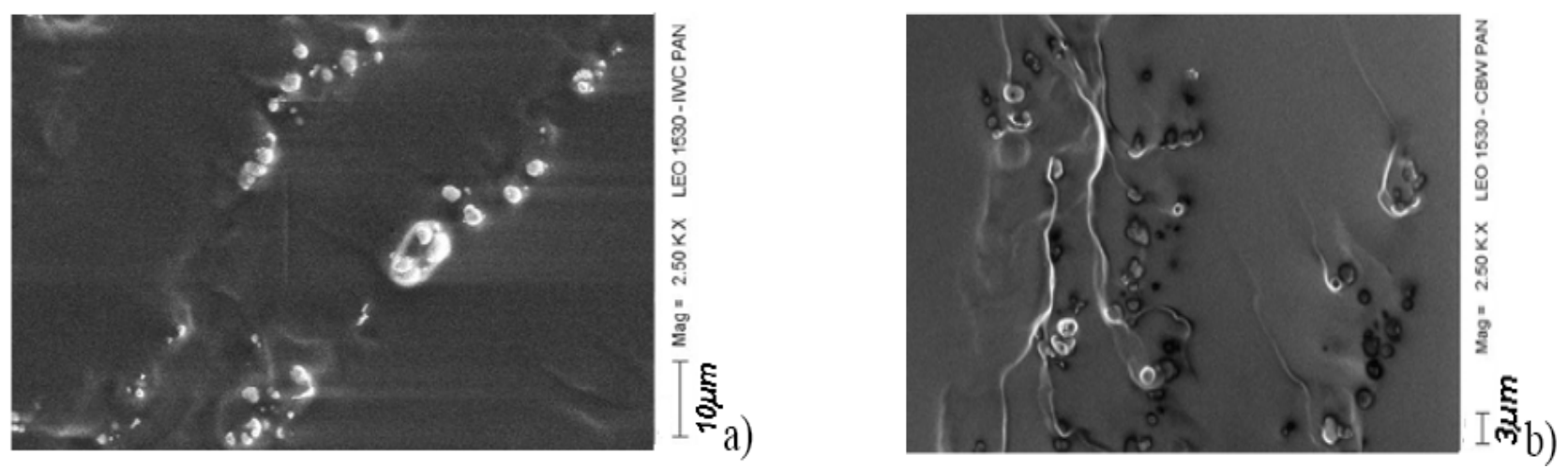

Fig.2. SEM images of MRE obtained from PU 80/20 filled with 11.5 vol. \% of carbonyl-iron particles, cured under magnetic field of a) $100 \mathrm{mT}$, b) $300 \mathrm{mT}$ (Kallio et al., 2003). 


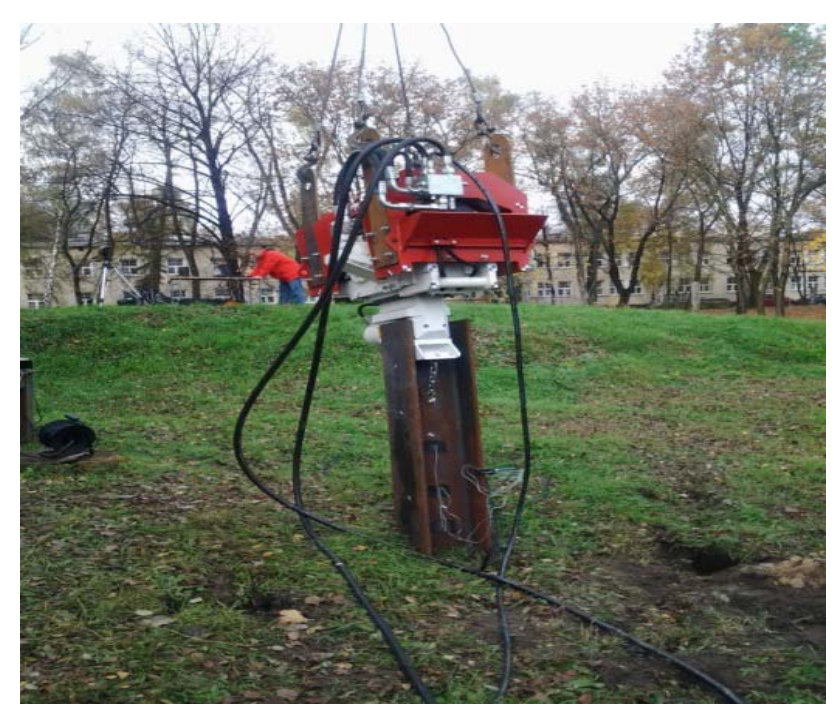

Fig.3. Experimental testing stage.

Different elastomers and magnetic particles can be used for fabrication of MREs. A strong external magnetic field is applied during the polymer curing process. The field induces dipole moments within the particles which relax into minimum energy states. The particle chains with collinear dipole moments are formed and the curing of the polymeric host material locks the chains in place. The particles can form separate chains, three-dimensional structures consisting of individual chains or more complex structures in which the particles have multiple interaction points (Michalczyk and Cieplok, 1999). The microstructures thus obtained determine the magnetorheological properties of the composites.

Applications of MR elastomers include automotive bushings and engine mounts, where significant changes in spring constant due to the applied magnetic field can be used to control stiffness and damping properties dynamically. Some examples of the MRE applications are presented in Fig.4.

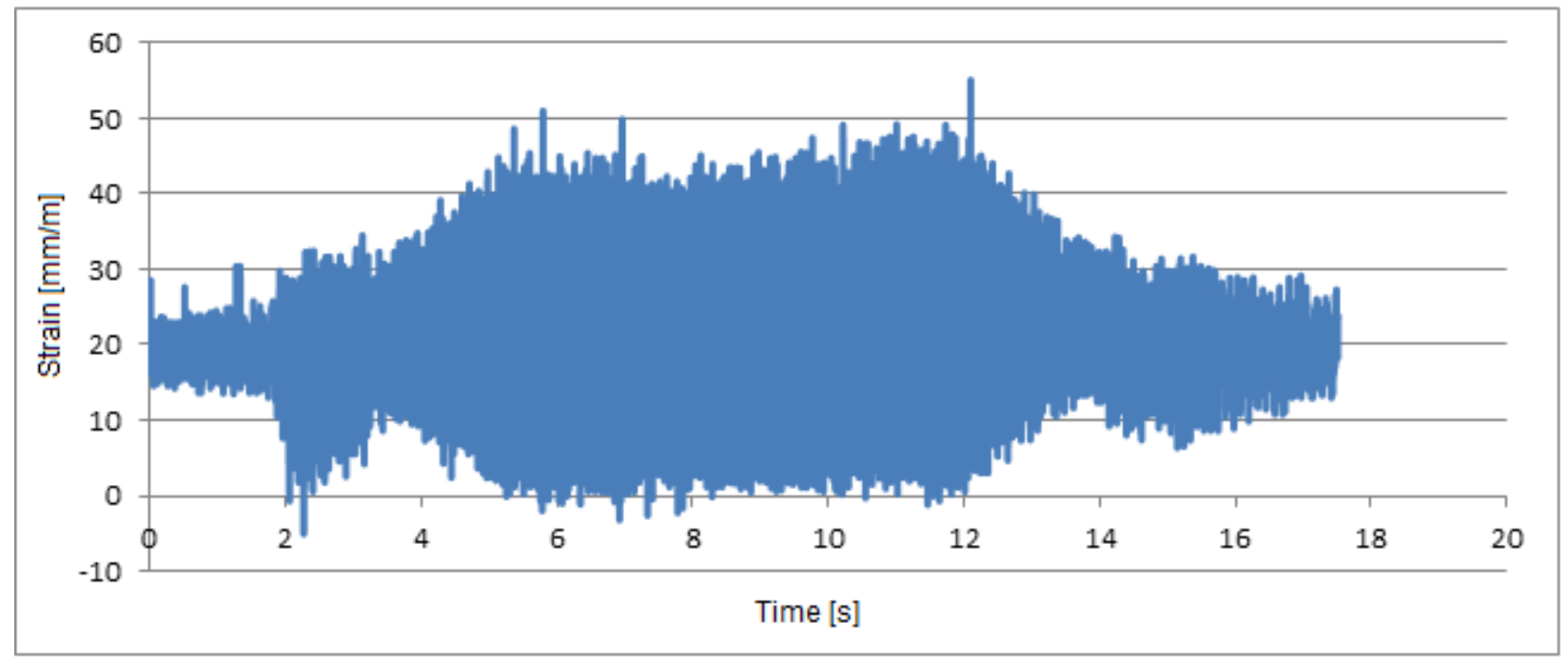

Fig.4. Filtrated signal from the 2nd extensometer. 
The work presented in the paper is a part of the modified VPH construction design process. It concerns the experimental research on the vibrations during the piling process and the analytical analyses of the gained signal. The results will be applied in the VPH control system.

\section{Experimental research}

The experimental research of vibrations that appear during the soil and VPH cooperation while piling was carried out. The VPH generates a wide range of different signals of various frequencies. The researched signal was registered with the use of two extensometers fixed on the pile: $1^{\text {st }}$ one - at the distance of $80 \mathrm{~cm}$ from the top edge of the pile, $2^{\text {nd }}$ one - at the distance of $130 \mathrm{~cm}$ from the top edge of the pile (the pile total height was $320 \mathrm{~cm}$ ). The signal was registered on the ESAM Traveller CF bridge amplifier- and data acquisition system. The testing stage was presented in Fig.5.

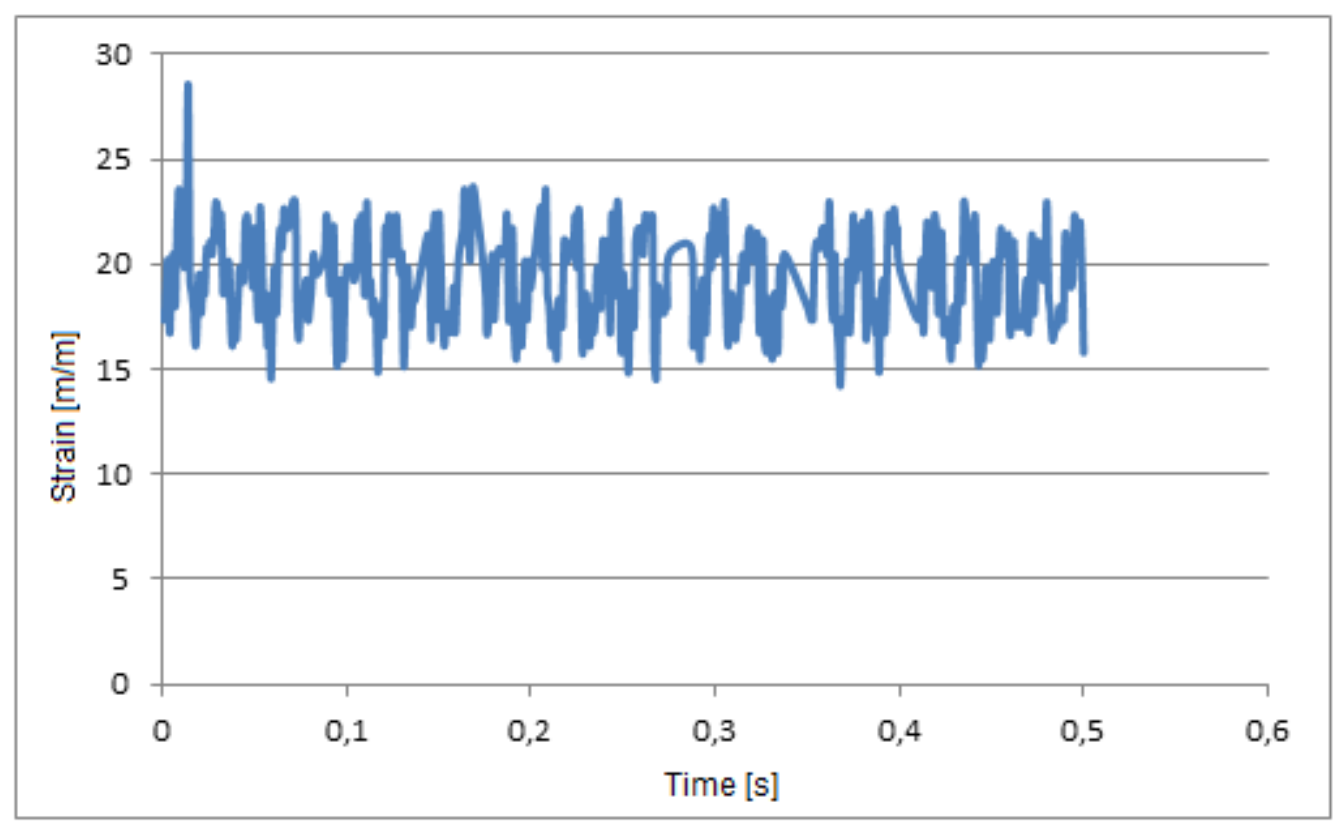

Fig.5. Filtrated signal from the 2nd extensometer for the time period from 0 to $0.5 \mathrm{~s}$.

The signal was registered in three tests. The gained signal is not a "clear" sinusoid, but a system of many components. It means that the cyclic deformation waves from VPH pile hitting are coupled with the waves reflected from the soil.

\section{Analytical study}

The signal analysis on the basis of the presented experiment results is very difficult, because the steel pile is an elastic element and gives a lot of additional frequencies. They have to be "separated" from the signal from VPH.

The first step to analyze the signal was an observation of time waveform for checking the frequency values vs. time. The second extensometer signal was filtrated with the use of Excel. The filtration process made it possible to remove the high frequency caused by the hitting pile. The resultant curve was shown in Fig.6.

Different work periods are visible on the chart. To show it the periods of $0.5 s$ were presented. The first period from 0 to $0.5 \mathrm{~s}$ is shown in Fig.7. The high frequencies are visible - vibration period of $0.024 \mathrm{~s}$, frequency $f=42 \mathrm{~Hz}$. It is a frequency for maximum angular velocity of VPH -2500 rotations/min, what gives 


$$
\omega=\frac{\pi n}{30}=\frac{2500 \pi}{30}=83, \quad 3 \pi=262 \frac{1}{\mathrm{~s}}
$$

and

$$
f=\frac{\omega}{2 \pi}=\frac{83,3 \pi}{2 \pi}=83, \quad 3 \pi=41,65 \mathrm{~Hz}
$$

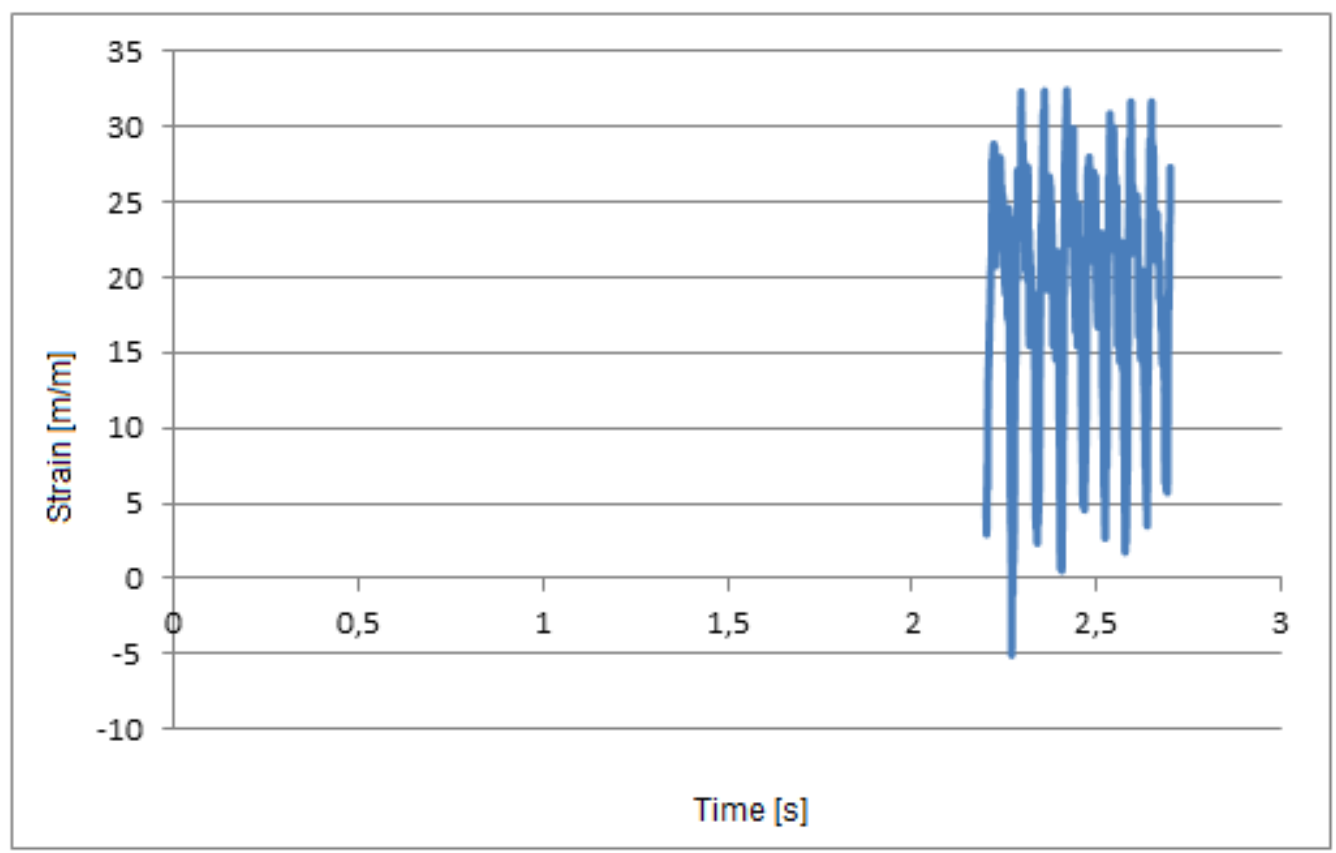

Fig.6. Filtrated signal from the 2nd extensometer for the time period from 2.3 to $2.8 \mathrm{~s}$.

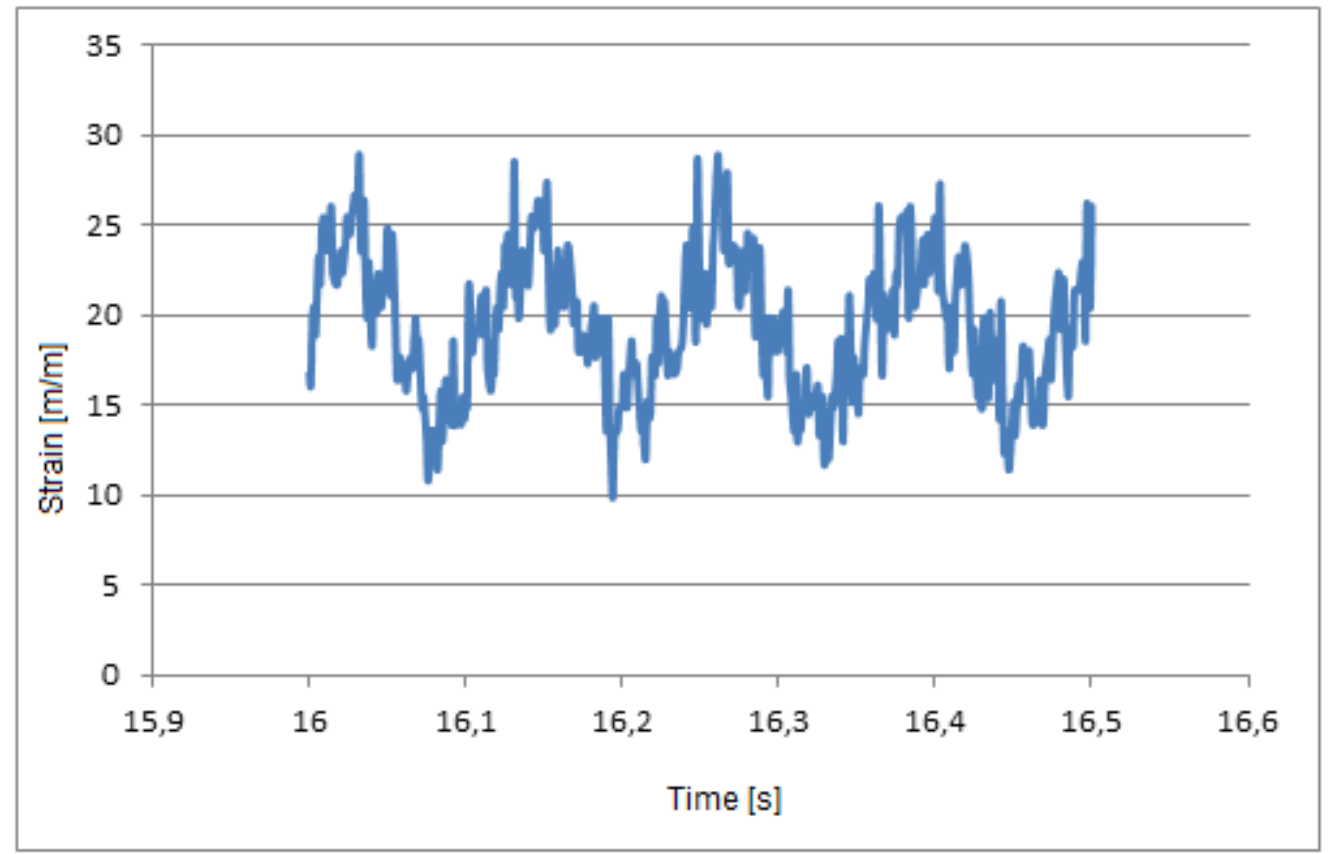

Fig.7. Filtrated signal from the 2nd extensometer for the time period from 8 to $8.5 \mathrm{~s}$. 
The next period from 2.3 to $2.8 \mathrm{~s}$ is presented in Fig.8. The vibrations period is $0.063 \mathrm{~s}$, and the frequency is much lower $-15 \mathrm{~Hz}$.

Finally, the period from 8 to $8.5 s$ is presented in Fig.9. Here, the vibrations period is $0.023 s$, and the frequency is very high $-43 \mathrm{~Hz}$.

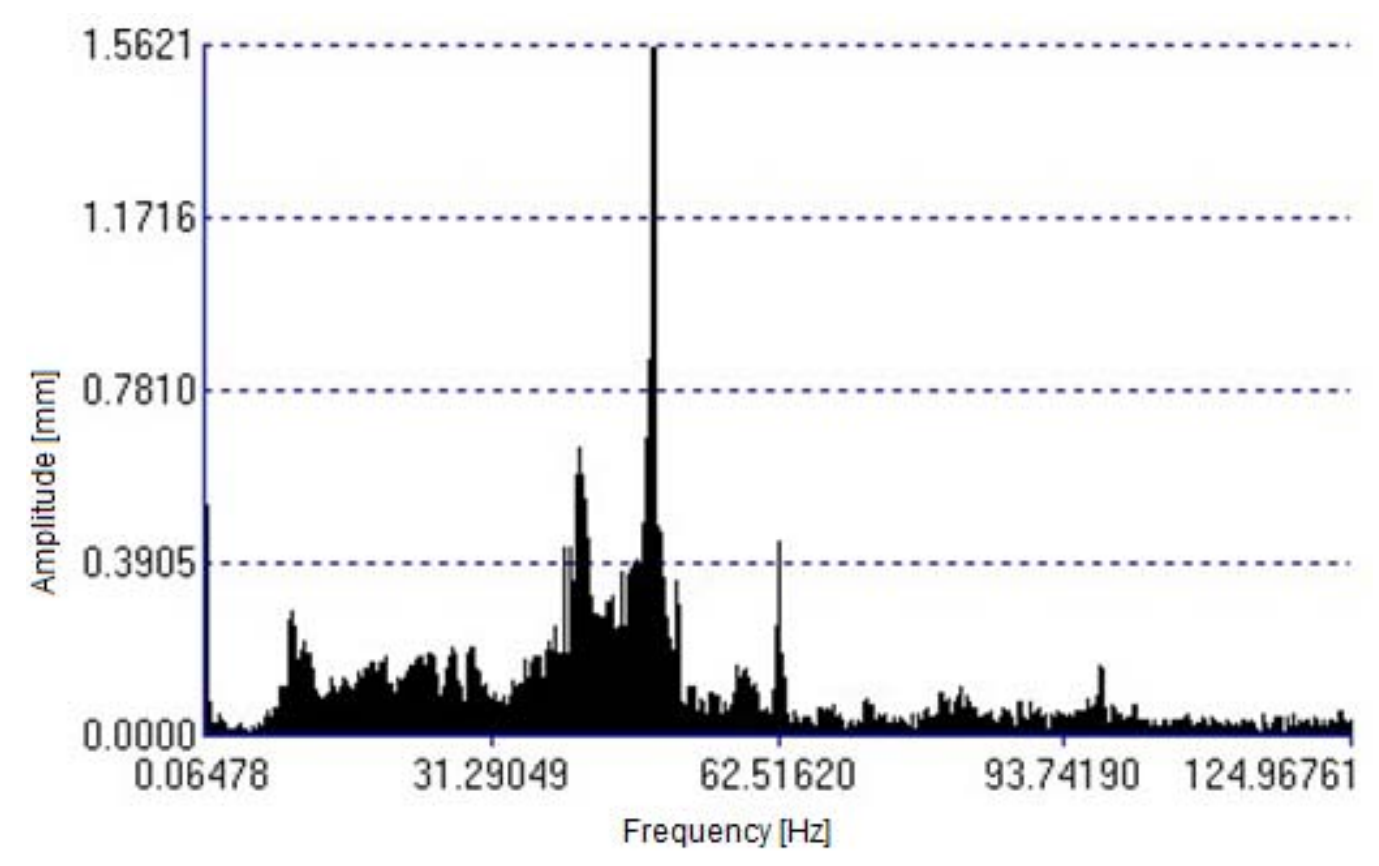

Fig.8. FFT analysis results - sum 14 components.

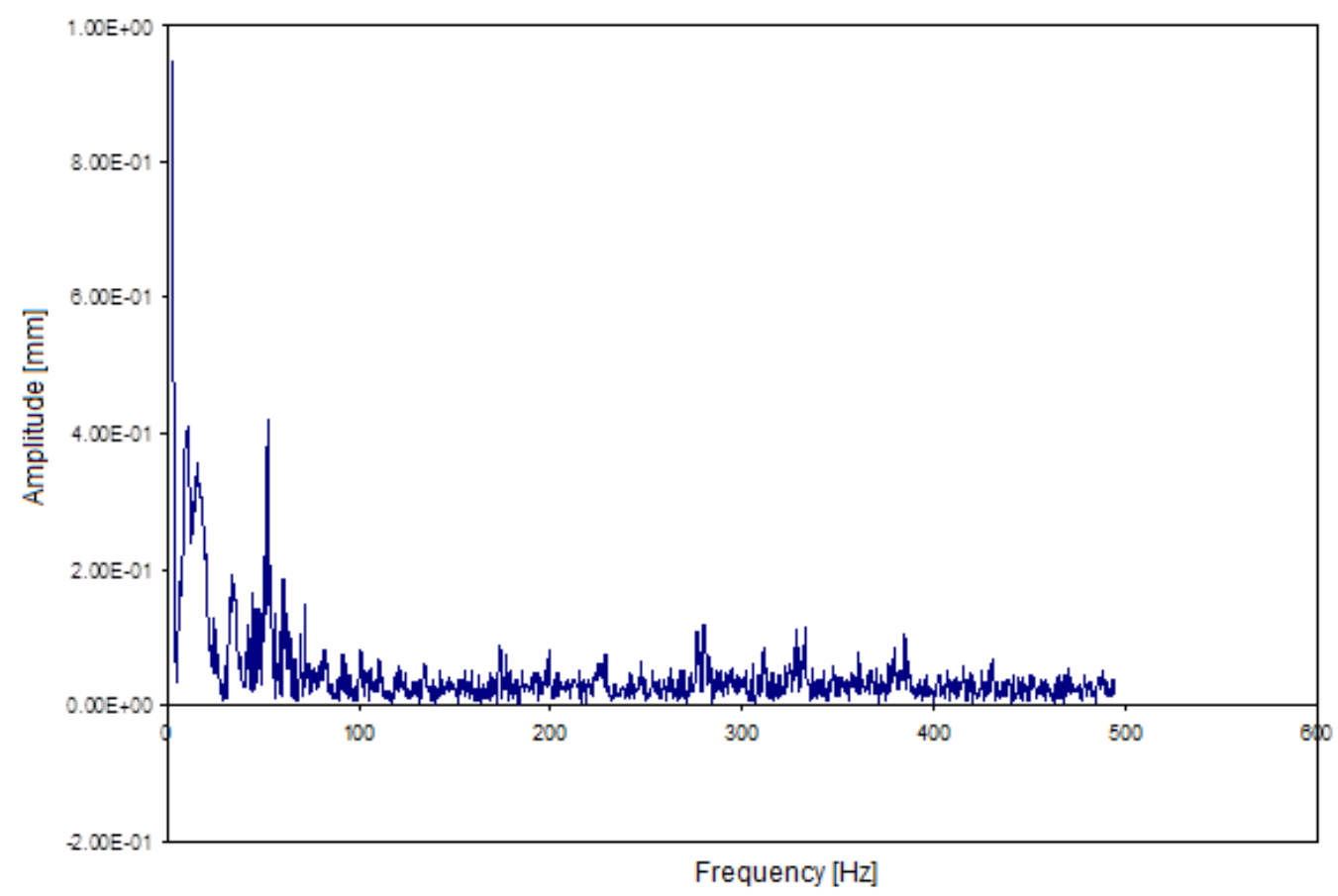

Fig.9. FFT analysis results - sum 13 components. 
On the basis of these initial analyses it can be concluded that during most of the time periods the VPH works with the high frequency - about $42 \mathrm{~Hz}$, which is a value for the maximum angular velocity and of course over resonance.

For more accurate analyses the Fast Fourier Transform was applied to the signal description. The fast Fourier transform (FFT) is an algorithm to compute the discrete Fourier transform (DFT) and its inverse. Fourier analysis converts time (or space) to frequency and vice versa; FFT rapidly computes such transformations by factorizing the DFT matrix into a product of sparse (mostly zero) factors [4]. As a result, fast Fourier transforms are widely used for many applications in engineering, science, and mathematics. The basic ideas were popularized in 1965, but some FFTs had been previously known as early as 1805 . Fast Fourier transforms have been described as "the most important numerical algorithms of our lifetime".

There are many different FFT algorithms involving a wide range of mathematics, from simple complex-number arithmetic to group theory and number theory. In the paper the following sum (Michalczyk and Cieplok, 1999) was used

$$
A(t)=A_{0}+\sum_{i=1}^{N} A_{i} \sin i \omega t
$$

where $A$ is an amplitude and $\omega$ is an angular speed.

The results of FFT analyses are presented in Fig.10 (sum 14 components) and Fig.11 (sum 13 components).

The results presented herein are taken from the same test and show highly nonlinear vibrations. It can be also marked that a high frequency of $48 \mathrm{~Hz}$ and amplitude of 0.5 dominates what shows the over resonance character VPH work. Also, the lower frequency appears when VPH goes through resonance.

\section{Summary}

1. On the basis of results achieved the vibration characteristics of VPH-pile-soil system were described.

2. Almost all vibration periods of the system have high frequencies $-42 \mathrm{~Hz}$, so it is a value for the maximum angular velocity and over resonance. Also, lower frequency appears - it will be regulated with MRE element application.

3. The results will be used for design of the MRE regulation system.

\section{Acknowledgements}

The paper was supported by a grant ID 18484 (Agreement No PBS1/A5/5/2012), financed in the years 2012-2015 by The National Centre for Research and Development, Poland.

\section{References}

An Y. and Shaw M.T. (2003): Actuating properties of soft gels with ordered iron particles: basis for a shear actuator.Smart Materials and Structures, vol.12, pp.157-163.

Kallio M., Aalto S. and Lindroos T. (2003): Preliminary test on a MRE device. - AMAS Workshop on Smart Materials and Structures SMART '03, mat. konf. pp.53-60. 
Boczkowska A. and Awietjan S. (2009): Magnetorheological urethane elastomers activated by magnetic field. POLIMERY 2009, vol.54, pp.26-30.

Fast Fourier Transforms, Connexions online book edited by C. Sidney Burrus, with chapters by C. Sidney Burrus, Ivan Selesnick, Markus Pueschel, Matteo Frigo, and Steven G. Johnson, 2008.

Michalczyk J. and Cieplok G. (1999): Highly effective systems for vibroisplations and vibrations reduction. Collegium Colombinum, Krakow.

Received: July 7, 2014

Revised: January 27, 2015 